Les jeux qui bifurquent : narration non-linéaire et spatialisation de l'intrigue. Le cas du Tactical RPG

\title{
Sébastien Wit
}

\section{OpenEdition}

\section{Journals}

Édition électronique

URL : http://journals.openedition.org/sdj/1022

DOI : $10.4000 /$ sdj. 1022

ISSN : 2269-2657

Éditeur

Laboratoire EXPERICE - Centre de Recherche Interuniversitaire Expérience Ressources Culturelles Education

\section{Référence électronique}

Sébastien Wit, «Les jeux qui bifurquent : narration non-linéaire et spatialisation de l'intrigue. Le cas du Tactical RPG », Sciences du jeu [En ligne], 9 | 2018, mis en ligne le 07 juin 2018, consulté le 01 mai 2019. URL : http://journals.openedition.org/sdj/1022 ; DOI : 10.4000/sdj.1022

Ce document a été généré automatiquement le 1 mai 2019.

Tous droits réservés 


\title{
Les jeux qui bifurquent : narration non-linéaire et spatialisation de l'intrigue. Le cas du Tactical RPG
}

\author{
Sébastien Wit
}

1 Ce que l'on nomme Tactical RPG (T-RPG), ou encore « jeu de rôle tactique » en français, est un genre vidéoludique qui naît au tout début des années 1990. Certes, le système de jeu sur lequel il est bâti n'est pas totalement inédit. Dès les débuts du jeu vidéo existent des systèmes de jeu utilisant un espace graphique isométrique sur lequel le joueur doit déplacer ses unités. Néanmoins, il faut vraiment attendre les années 1990 pour que ce type de jouabilité (gameplay) devienne la pierre angulaire d'une catégorie générique à part entière. C'est pourquoi on considère généralement le premier opus de la série Fire Emblem comme l'ancêtre historique du T-RPG actuel. Développé pour la Nintendo Entertainment System (NES) par Intelligent Systems et distribué par Nintendo en 1990 (au Japon uniquement), Fire Emblem: Ankoku Ryū to Hikari no Tsurugi (« Fire Emblem : le dragon des ténèbres et l'épée de lumière ») pose les fondements du T-RPG et, par là même, d'une forme spécifique de narrativité.

2 En tant que mélange entre jeu de rôle et jeu de stratégie au tour par tour, le T-RPG apparaît comme un objet hybride devant concilier la tension narrative propre au jeu de rôle et le fonctionnement purement a-diégétique des échecs. En effet, à bien des égards, les échecs sont le modèle emblématique du jeu de stratégie au tour par tour. Aux échecs, chaque joueur déplace - l'un après l'autre - ses pièces afin de l'emporter sur l'adversaire. Tout comme leurs prédécesseurs analogiques (notamment la série des Tactics d'Avalon Hill Game Company), les premiers jeux de stratégie au tour par tour sur support électronique s'inscrivent dans la lignée de la pratique échiquéenne, tout en faisant de l'espace de jeu un véritable environnement. Alors que la surface de l'échiquier n'est jamais qu'un quadrillage abstrait, un jeu vidéo comme Empire: Wargame of the Century de Nothwest Software (première version: 1977) transforme la grille ludique en un morcellement concret; chaque case de l'univers du jeu dispose d'attributs physiques (telle case est un morceau d'océan, telle autre un fragment de plaine, etc.). Autrement dit, 
quel que soit son médium, le jeu de stratégie (wargame) se caractérise par la consistance topographique qu'il donne à l'antique plateau de jeu, et c'est précisément cela que l'on va retrouver dans le $T-R P G$. De ce fait, dans la suite de cet article, j'analyserai la manière dont le système de jeu du T-RPG reprend à son compte cette mise en géographie de l'espace isométrique afin d'en faire le cœur d'une dynamique narrative.

\section{Petite histoire du T-RPG}

\section{J-RPG et T-RPG face au RPG occidental}

3 Né au Japon, le T-RPG ne peut se comprendre qu'en lien avec ce que l'on désigne généralement comme le J-RPG (le jeu de rôle japonais), par opposition aux RPGs occidentaux. Prenant place au sein d'un univers médiéval fantastique, le premier Fire Emblem mobilise un univers fictionnel déjà bien éprouvé par les J-RPGs apparus entre 1985 et 1990. De plus, comme rappelé en introduction, le jeu de stratégie au tour par tour n'est pas une invention du T-RPG. En Occident, des jeux comme Ultima III d'Origin Systems (1983) proposent dès le début des années 1980 un système de jeu mélangeant gestion d'un groupe de personnages, phases d'exploration et phases de combat. ${ }^{1}$

4 L'une des grandes innovations d'Ultima III réside dans son système de combat: le joueur dispose de plusieurs personnages qu'il peut déplacer à sa guise. Par ces déplacements tactiques intégrés à la phase de combat, Ultima III se démarque d'un autre grand RPG développé par Sir-Tech au début des années 1980: Wizardry: Proving Grounds of the Mad Overlord (1981), premier titre de la série des Wizardry. Dans ce jeu, le joueur contrôle également un groupe de personnages, mais la phase de combat se limite à la succession des actions effectuées par les différents protagonistes. En l'absence d'un champ de bataille proprement dit, le joueur de Wizardry ne prend aucune décision quant au positionnement des unités. Cette différence entre le système de combat des deux jeux s'explique aisément du point de vue de la conception de jeu (game design). Pour la première fois dans l'histoire du RPG, les combats d'Ultima III ne se déroulent plus dans le même écran que celui qui est dédié à l'exploration. Séparé, cet écran de combat donne au jeu un aspect tactique plus développé. Une grande partie de la réussite du joueur dépend alors de la disposition spatiale des personnages, en mouvement sur un espace isométrique.

5 Aujourd'hui encore, l'influence d'Ultima III sur les séries historiques du J-RPG que sont Dragon Quest (Enix, 1986) et Final Fantasy (Square Co., 1987) demeure un point central dans les polémiques - souvent vaines - autour de l'opposition entre RPGs japonais et occidentaux (Stern, 2012) ; selon certains cercles de fans, les séries les plus populaires du $J-R P G$ se seraient contentées de reprendre des concepts de jeu au RPG occidental. ${ }^{2}$ Pourtant, si le J-RPG semble emprunter un certain nombre d'éléments de la jouabilité d' Ultima III, il laisse délibérément de côté l'aspect positionnel de son système de combat. C'est le T-RPG qui va s'emparer de ce mécanisme et en faire un élément constitutif de son mode de jeu.

\section{La phase de combat : lieu de la narrativité du T-RPG}

6 Pour se rendre compte de ce que le T-RPG emprunte au jeu de rôle occidental et de la manière dont il s'en distancie, on peut commencer par examiner leurs systèmes de 
combat respectifs. Si le premier Fire Emblem se fonde lui aussi sur un système de gestion de plusieurs personnages, le nombre de ces derniers est beaucoup plus important que dans Ultima III. Face aux quatre personnages jouables du RPG de 1983, Fire Emblem en propose une cinquantaine! Même s'il n'est jamais possible de tous les déployer en même temps, le dernier niveau du jeu permet d'en utiliser quinze. Pareillement, en ce qui concerne le mouvement des personnages, chaque unité d'Ultima III ne peut se déplacer que d'une case par tour, alors que Fire Emblem institue des possibilités de déplacement différenciées pour chaque unité (qui dispose donc d'une statistique de mouvement personnelle).

7 De plus, bien qu'Ultima III présente un espace de combat différencié (avec des carrés correspondant à des zones de forêt, etc.), le terrain ne dispose pas de propriétés intrinsèques contrairement à ce que l'on trouve dans Fire Emblem. Les différentes cases y influent sur les statistiques de mouvement, de précision, d'esquive, etc. Dans Ankoku Ryū to Hikari no Tsurugi, une case "forêt » donne, entre autres, un bonus d'évasion de $15 \%$ ainsi qu'un malus de mouvement (ce dernier étant plus élevé pour les archers et les unités à cheval). ${ }^{3}$ Ce degré de précision dans les attributs du terrain montre bien l'aspect central occupé par celui-ci dans le système de jeu. En cela, la caractérisation topographique de l'espace apparaît bel et bien comme une spécificité du T-RPG, comme son principal ajout en comparaison d'un espace isométrique ne remplissant qu'une fonction d'illustration dans des jeux de rôle comme Ultima III.

Par ailleurs, à la différence des J-RPGs, Fire Emblem fait complètement disparaître la phase d'exploration, - pourtant traditionnelle du jeu de rôle. À aucun moment le joueur ne peut acheter de l'équipement en ville ou parcourir des donjons. L'espace de jeu est uniquement constitué des différents champs de bataille. Tout ce qui concerne le ravitaillement en armes ou encore les conversations entre les personnages se trouve intégré au déroulement du combat. Construit comme une phase de combat perpétuel, Fire Emblem transforme tous les éléments de sa diégèse en événements tactiques. Le joueur doit effectuer toutes les actions du jeu au cours de cette seule phase. Parce qu'Ultima III ou Dragon Quest sont des jeux où l'histoire se déploie en dehors des moments de combat, on comprend mieux en quoi la narrativité du T-RPG diffère grandement de celle des RPGs précédents.

9 Cette grande spécificité narrative correspond à la raison pour laquelle on assiste, à la suite de Fire Emblem : Ankoku Ryū to Hikari no Tsurugi, à une période d'expérimentation qui va durer jusqu'en 1995 environ. Entre 1990 et la parution de jeux comme Der Langrisser (Nippon Computer Systems, 1995) ou le célèbre Tactics Ogre: Let Us Cling Together (Quest, 1995 également), le genre naissant du T-RPG va explorer les potentialités narratives que lui laisse un système de jeu a priori restreint à son aspect tactique.

\section{La map : l'unité narrative du T-RPG}

\section{Précision lexicale}

10 Sur le plan de la narratologie, la construction du récit des T-RPG repose presque exclusivement sur ce que l'on peut nommer des maps. On préférera ici le terme anglais au français « cartes ", bien qu'il s'agisse généralement de la traduction littérale associée. Mis à part lorsque l'on parle de cartes à jouer, le substantif carte ne dispose pas vraiment d'un sémantisme ludique en français. La plupart du temps, lorsque l'on parle du jeu vidéo, on a 
plutôt tendance à utiliser le terme «niveau " pour désigner une portion de l'espace ludique. Cependant, parler de niveaux renvoie davantage au genre du jeu de plateforme dans lequel l'aspect géographique ne joue pas un rôle aussi central que dans le T-RPG. Or, l'anglais map présente l'avantage de désigner tout à la fois la structure spatiale d'un jeu, ainsi que la vision topographique qui est celle du T-RPG. En outre, cela permettra de réserver le terme « carte » à la carte du monde de certains jeux, par opposition aux maps.

\section{Le T-RPG et ses ellipses, ou la prédominance de la disruption chronologique}

11 Cette parenthèse lexicologique refermée, on peut noter que la structure en maps du T-RPG en fait un excellent paradigme de ce que Henry Jenkins (2002, p. 121) nomme des espaces producteurs de récit (narratively compelling spaces). Dans ces « histoires spatiales» (" spatial stories »), le voyage à travers l'espace remplace la diégèse traditionnelle. Si l'on veut résumer l'intrigue de Fire Emblem : Ankoku Ryū to Hikari no Tsurugi, il ne s'agit pas tant de la geste héroïque de Marth que de sa traversée d'un ensemble de territoires, de la petite île de Talis au château de Medeus en plein cœur de Durhua. Fragmenté en zones de combat, le récit perd une grande part de sa dimension causale et chronologique au profit d'une micronarrativité faite d'événements scriptés, déclenchés via les interactions entre les unités du joueur et des éléments spécifiques inscrits au sein des grilles isométriques (personnages, lieux, etc.). Si, comme le déclare Jenkins (2002, p.125), une telle micronarrativité est propre au médium vidéoludique en lui-même, le $T$ - $R P G$ se démarque par la place prépondérante qu'occupent les ellipses au sein de sa structure narrative globale.

Pour reprendre l'exemple d'Ankoku Ryū to Hikari no Tsurugi, sur le plan de la fabula (l'histoire racontée), le passage d'une map à une autre suppose un déplacement des troupes de Marth à travers le continent. Or, le jeu ne disposant d'aucune phase d'exploration, ces mouvements ne sont jamais vécus par le joueur. Ce dernier se trouve donc simplement téléporté d'un endroit à un autre sans que la chaîne chronologique du récit ne soit saturée. Ainsi, entre le scénario 3 et le scénario 4 d'Ankoku Ryū to Hikari no Tsurugi, le joueur passe-t-il directement des montagnes des Dents de la Goule aux plaines du royaume d'Aurelis. Si le texte liminaire du scénario 4 permet de reconstituer la chaîne causale, il n'en demeure pas moins que le récit subit une disruption chronologique ayant pour effet de couper le joueur d'une séquence diégétique (en l'occurrence, la sortie des montagnes). En cela, la structure narrative du T-RPG se place dans la tradition du wargame et de sa construction en scénarios isolés, eux-mêmes inscrits au sein d'une « campagne » servant de fil rouge narratif.

\section{Le wargame : ancêtre de la narrativité du T-RPG}

En ouverture de cet article, j'ai déjà rappelé la parenté entre la configuration spatiale du $T-R P G$ et celle des wargames. Or, le lien unissant ces deux genres ludiques n'est pas simplement d'ordre visuel. Ce sont véritablement les wargames qui inventent la diégèse spatialisée que l'on retrouve dans les T-RPG. Dans son travail sur le legs des wargames au jeu de rôle à la Donjons \& Dragons, Jon Peterson (2012) revient sur la fonction essentielle que revêt pour le genre la différence entre aspect stratégique et aspect tactique. L'aspect stratégique renvoie aux mouvements des troupes sur le champ de bataille. Circonscrit à 
un événement précis assigné à un cadre spatio-temporel restreint, l'aspect tactique s'oppose à l'architecture globale de l'aspect stratégique. Système de jeu dont l'exemple emblématique demeure Risk (Parker Brothers, 1959), la stratégie requiert une macrogouvernance des diverses unités. Il s'agit de les faire se déplacer à travers de vastes zones géographiques, et ce sur une période de temps étendue. Dans le wargame, l'articulation entre ces deux niveaux se fait justement par le biais de la notion de campagne. Peterson (2012, p. 49) analyse notamment le cas de la campagne hyborienne de Tony Bath. Élaborée dans les années 1950 d'après l'univers fantastique (fantasy) de Conan le barbare, le personnage de Robert E. Howard, l'Hyborian Campaign comprend une carte du monde sur laquelle se déplacent les différentes troupes. Le lieu de la rencontre sur la carte détermine la map du combat. Dans ce cas précis, Bath conçoit un système de jeu où la géographie globale est garante de l'unité narrative des différents moments tactiques.

la perspective de Peterson, le jeu de rôle moderne - auquel appartient le T-RPG - se constitue à partir de systèmes de jeux empruntés au wargame. D'une part, Donjons \& Dragons reprend les espaces quadrillés que l'on trouvait dans la série des Tactics d'Avalon Hill Game Company. D'autre part, à la suite de la campagne «Blackmoor» de Dave Anerson, le RPG à la Donjons \& Dragons se fonde sur une continuité entre différents scénarios, se déroulant chacun sur une map spécifique. C'est précisément cette continuité qui permet le développement et l'évolution des personnages des joueurs. De ce fait, en empruntant directement des éléments de jouabilité au genre du wargame, le T-RPG renoue à la fin des années 1980 avec l'entreprise d'hybridation originelle par laquelle naît le genre du RPG au début des années 1970. Le T-RPG n'est pas le premier à faire de la map un ressort narratif. Il s'inscrit dans une tradition ludique que l'on peut faire remonter à la seconde moitié du XIX siècle.

\section{La saturation du récit, ou la narration aux prises avec l'axiomatique du jeu}

Après cette brève présentation de l'histoire du wargame et de l'influence de son système de jeu sur le T-RPG, nous pouvons revenir du côté du jeu vidéo. Quand on parle de saturation du récit vidéoludique, il faut entendre la coïncidence temporelle entre le temps de la fabula et celui qui est effectivement vécu par le joueur, autrement dit le temps de l'axiomatique. ${ }^{4} \mathrm{Si}$ la seule distinction entre la fabula (l'histoire racontée) et le sjuzhet (le récit de cette histoire) s'adapte bien aux formes de récit ne comportant pas une dimension axiomatique, elle n'est plus suffisante lorsque le temps du récit se mêle à celui - réel - expérimenté par le joueur. ${ }^{5}$ D'une certaine manière, dans le jeu vidéo, ce que le joueur ne réalise pas par lui-même se trouve déplacé à l'extérieur de l'expérience de jeu, mis en marge du récit qui perd donc en saturation. Ainsi, la saturation du récit correspond-elle au mécanisme par lequel le versant axiomatique du jeu vidéo tend à incorporer au maximum les divers pans du récit au temps de jeu.

Afin de favoriser cette saturation du récit, les T-RPG ont rapidement tenté de renforcer leur dispositif narratif. Sorti en 1992, Fire Emblem Gaiden constitue la suite directe d' Ankoku Ryū to Hikari no Tsurugi. Malgré un système de jeu similaire, ce second volet de la série se distingue par l'existence d'un écran d'exploration sur lequel le joueur déplace les deux personnages principaux afin de visiter différents lieux : villages, châteaux, donjons, etc. À travers cette carte du monde navigable, le joueur peut lui-même faire le lien entre les différents champs de bataille. Bien qu'il n'y ait pas de narration explicite à propos des 
mouvements d'Alm et de Celica, le joueur vit bel et bien chacun des déplacements. Il les décide lui-même, ce qui restaure une forme de continuité dans l'expérience de jeu. Il n'est pas anodin que le genre naissant du T-RPG trouve ainsi dans son ancêtre du wargame des mécanismes susceptibles d'assurer sa continuité narrative. Grâce à la carte du monde, Fire Emblem Gaiden met en scène une globalité spatiale, celle de l'aspect stratégique propre à la macrogouvernance du wargame. Cependant, afin de favoriser la saturation du récit, Gaiden ne se contente pas de reprendre au wargame des éléments de jouabilité Outre la scission du monde en deux itinéraires, la carte de Gaiden s'accompagne d'un compteur de tours faisant office de marqueur temporel des décisions du joueur. Parce qu'il est automatiquement enregistré dans le temps du jeu (avantage de l'électronique par rapport à l'analogique), chaque déplacement constitue réellement un temps de jeu. Ici, la distinction entre le temps $d u$ jeu et le temps de jeu a son importance. Alors que le temps de jeu renvoie à la temporalité vécue de l'expérience ludique, le temps $d u$ jeu correspond à la manière dont le jeu vidéo instaure sa propre temporalité. Le temps $d u$ jeu est à michemin entre le temps du récit et celui de l'axiomatique. En faisant du temps du jeu l'équivalent d'une horloge interne, Fire Emblem Gaiden déplace la fonction de saturation du récit; elle ne pèse plus sur le temps de la fabula mais sur celui du jeu.

Alors que le système de carte du monde sera abandonné dans la suite de la série des Fire Emblem (jusqu'à The Sacred Stones en 2004), on le retrouve dans le jeu Tactics Ogre: Let Us Cling Together sorti en 1995. Comme dans Gaiden, le joueur y suit le fil du récit en passant d'un lieu à un autre. En revanche, les unités ennemies n'apparaissent pas sur la carte. Chaque lieu est représenté par un point : le joueur ne sait donc jamais ce qu'il va trouver en se rendant à tel ou tel endroit. Certes, Gaiden comportait déjà un mécanisme similaire. Durant les actes 3 et 4 , des ennemis supplémentaires peuvent apparaître aléatoirement au cours des déplacements des deux protagonistes. Cependant, ces ennemis restent visibles pour le joueur. Seuls leurs déplacements sur la carte du monde constituent une inconnue. Dans la mesure où le système de jeu laisse le joueur dans le mystère, on peut dire que Tactics Ogre dramatise davantage les intermèdes a-narratifs séparant chaque combat.

Par sa rhétorique procédurale, Tactics Ogre instaure entre les séquences de combat une forme de tension, que l'on ne saurait totalement assimiler à la tension narrative théorisée par Raphaël Baroni (2007), mais qui drape tout de même l'axiomatique ludique des mécanismes de la curiosité. Dans La Tension narrative, Baroni (2007, p. 267) distingue clairement la «mise en intrigue par la curiosité » de la «mise en intrigue par le suspense ». Selon lui, le propre de la curiosité est d'être étrangère à toute dimension temporelle. Déconnectée de l'action diégétique, la curiosité porte sur la structure globale du récit, tandis que le suspense n'a de sens que dans l'anticipation - toujours temporelle - de l'action à venir. Les renforts de Gaiden ou les combats aléatoires de Tactics Ogre correspondent à des événements extérieurs à l'intrigue. Ils n'ajoutent rien à l'enchaînement temporel du jeu. Ils servent surtout à remplir le passage du temps de jeu grâce à d'autres combats. Ces derniers étant le seul cadre de jeu du T-RPG, on comprend bien comment leur usage comme intermèdes a-narratifs permet paradoxalement de saturer le récit. Il s'agit de faire vivre en temps de jeu le passage d'un événement à un autre afin d'éviter au joueur l'impression de passer automatiquement d'un champ de bataille au suivant, d'un moment clé de l'intrigue à sa suite sans que rien ne se soit déroulé entre-temps. C'est en alternant les maps de temps forts (inscrites dans la diégèse) et d'entre-temps (en marge de la diégèse) que le T-RPG se voit en mesure de maintenir une chronologie du récit, et ce malgré une structure narrative totalement spatialisée. 


\section{La bifurcation, un mécanisme de re-temporalisation de l'intrigue}

\section{L'introduction de la bifurcation narrative dans le T-RPG}

19 Parmi les expérimentations du début des années 1990 portant sur la forme narrative du T$R P G$, l'introduction de bifurcations au sein du récit constitue un tournant majeur. Déjà esquissé dans le dédoublement narratif de Fire Emblem Gaiden entre les lignes diégétiques parallèles d'Alm et de Celica, ce passage à un système de jeu non-linéaire (nonlinear gameplay) illustre une volonté de fluidifier l'extrême spatialisation de l'intrigue induite par une architecture en maps. Au cours de l'année 1995, vont sortir deux jeux poussant au maximum la délinéarisation amorcée par le second opus de Fire Emblem. Ce sont Der Langrisser (juin 1995) et Tactics Ogre (octobre 1995), déjà mentionnés précédemment.

Je commencerai ici par m'arrêter sur le cas de Der Langrisser, généralement moins connu que Tactics Ogre (ne serait-ce qu'en raison du remake pour la PlayStation Portable de Sony dont Tactics Ogre a profité en 2011). Der Langrisser est une adaptation sur PC-FX de NEC et SNES de Langrisser II, sorti en 1994 sur la Genesis de Sega (c'est-à-dire un an plus tôt). Première chose à noter au sujet de Der Langrisser : les différences entre son système de jeu et celui - linéaire - de son prédécesseur. Par rapport à Langrisser II qui ne comportait qu'une seule ligne narrative, Der Langrisser propose neuf fins alternatives, venant conclure pas moins de quatre parcours diégétiques distincts. Il s'agit là de l'une des arborescences narratives les plus complexes de cette période du jeu vidéo !6 Au fil de l'intrigue, le joueur est amené à choisir - plus ou moins explicitement - entre différents camps. C'est donc à lui que revient la décision de se ranger aux côtés des combattants de la Lumière ou de s'allier aux troupes de l'Empire. Ce premier choix intervient au cours du scénario 7.

Cependant, il est à noter que le joueur ne peut rejoindre l'Empire qu'à la condition de ne pas avoir vaincu certains personnages au cours des scénarios 2 et 5 . Plus précisément, ni Laird (scénario 2), ni Zorum (scénario 5) ne doivent avoir été vaincus. Dans le cadre d'une partie normale, il est quasiment impossible de vaincre Laird dans le scénario 2, alors que Zorum est presque toujours éliminé. En effet, la conception du jeu (game design) attribue à ces deux unités des fonctions très différentes. D'un côté, Zorum attaque directement le joueur, et dispose d'un niveau proche des unités de ce dernier. De l'autre, dans le scénario 2, Laird est beaucoup trop puissant pour être vaincu normalement. Unité évoluée de niveau 8, celui-ci est bien supérieur au niveau normal des personnages du joueur en début de partie. De plus, étant une unité montée, il dispose d'un avantage tactique sur Loren, l'allié contrôlé par l'ordinateur qui vient en aide au joueur. Dès lors, il n'y a aucune raison pour que Laird soit vaincu au cours du scénario 2. Pourtant, le scénario propose bien deux conditions de victoire distinctes l'une de l'autre. Pour la première condition, il suffit au joueur de permettre à Liana de s'enfuir par le nord de la map. D'une façon tout à fait logique, il s'agit de la stratégie la plus facile à adopter. Alors que Loren retient les troupes de Laird (tout en sachant qu'il ne peut le faire indéfiniment en raison de son désavantage tactique), le joueur peut prendre la route du nord, tout en se débarrassant des renforts qui apparaissent au fil de sa progression. Toutefois, il est également possible de remporter le scénario en se débarrassant de toutes les unités de la map, Laird compris. Mais cela n'est souvent envisageable que si le joueur connaît suffisamment le jeu pour 
planifier la mort de Laird en amont. Autrement dit, à bien des égards, l'impossibilité d'accepter la proposition de Léon au scénario 7 ne survient jamais au cours d'une partie normale. Le joueur doit décider presque délibérément de ne pas pouvoir prendre le parti de l'Empire pour que cela se produise. Et, dans le cas où le joueur élimine par hasard Laird au cours du scénario 2 , il n'est alors pas censé savoir qu'en éliminant Zorum, il se fermera les portes d'une bifurcation narrative (dans la mesure où le choix ne lui sera jamais proposé).

Dans Der Langrisser, certains des ressorts ludiques de la bifurcation narrative se révèlent particulièrement opaques pour le joueur. Lorsque celui-ci décide de laisser Laird en vie, ce n'est pas par respect pour la cause de l'Empire. Hormis dans le cas où le joueur n'est pas un joueur naif, mais qu'il joue en ayant connaissance des scripts de Der Langrisser, ses actions ne résultent pas systématiquement d'un choix éclairé. Pris dans l'axiomatique du système ludique, le joueur ne se trouve jamais confronté à un vrai dilemme moral (Lavocat, 2016). Recommencer une partie revient à se donner la chance d'agir autrement, de procéder à un choix différent et d'explorer un autre possible narratif - c'est-à-dire un mode de jeu différent, prenant place sur des maps différentes. Derrière le mécanisme de la bifurcation, le jeu se scinde spatialement entre deux chemins, deux itinéraires à travers le monde fictionnel. Dans le T-RPG, la narrativité n'est jamais autonome. Comme dans le wargame, elle est constamment annexée par l'architecture spatialisée de la trame ludique: toute ligne diégétique (ou campagne) n'existe qu'à travers la succession des maps qui la composent.

\section{De la map à la logique du récit}

L'exemple de Der Langrisser illustre parfaitement la manière dont la bifurcation fait entrer perpétuellement en collision la non-linéarité du système de jeu, et celle de la narrativité. C'est l'articulation entre ces deux niveaux qu'il convient à présent d'examiner afin de pouvoir dégager un modèle théorique de la bifurcation dans le T-RPG.

Lorsqu'au scénario 7 le joueur de Der Langrisser fait son choix entre rester dans le camp des descendants de la Lumière, ou se ranger du côté de l'Empire, cela va d'abord se manifester dans son parcours spatial sur la carte du monde. Dans les deux cas, le scénario 8 se déroule dans les montagnes proches de Reitel, sur un pont suspendu que les troupes d'Elwin doivent parvenir à franchir. Quelle que soit la voie choisie ("Lumière » ou "Empire »), l'objectif d'Elwin ne change pas: il désire obtenir l'épée légendaire Langrisser qui se trouve dans le sanctuaire de Baldea. Du pont suspendu au temple de Baldea, les scénarios 8 à 11 de chacune des voies ont un même point de départ et un même point d'arrivée. La seule différence est dans le trajet parcouru pour parvenir à destination. Dans la voie «Lumière ", Elwin se dirige vers l'est à la sortie des montagnes, alors qu'il bifurque vers l'ouest dans la voie "Empire». Cette séparation est/ouest se traduit par des différences entre les maps des scénarios 9 et 10 des deux voies. Bien que l'histoire demeure peu ou prou inchangée, quand on examine ces maps dans le détail, on constate que ces dernières servent de support à la dramatisation du choix du joueur, notamment dans le cas du scénario 9 de la voie «Empire ». Dans ce scénario, Elwin arrive dans un village où il va devoir faire face à Cherie, ancienne alliée et membre des descendants de la Lumière. Parce qu'il prend place dans un village, ce combat rappelle ceux des scénarios 1 et 6 dans lesquels Elwin devait protéger des civils. Or, dans ce scénario 9, c'est désormais Cherie qui organise l'évacuation des habitants afin de les 
protéger des exactions de l'Empire. Le fait que la map reprenne la géographie d'un hameau sert de support physique à la représentation du basculement moral du personnage d'Elwin, désormais passé du côté de l'oppresseur. En comparaison, le scénario 9 de la voie « Lumière » se déroule sur une map mettant en scène un fort militaire assiégé par les troupes de l'Empire, qui n'hésitent pas à instrumentaliser des civils afin de parvenir à leurs fins. Entre la map du hameau et celle du fort d'Aaron, ce sont bien deux intrigues différentes qui se déploient, mais uniquement au sein de la map. Chaque topographie se fait le miroir d'un affrontement des valeurs morales - affrontement découlant de l'issue du scénario 7. L'espace prend une dimension narrative : il raconte un conflit éthique. On voit bien ici la micronarrativité à l'œuvre. La topographie concrète de l'espace isométrique porte une dramatisation narrative restreinte à la map. Seule la mise en rapport de cette dernière avec les autres maps lui permet d'être le théâtre d'un moment narratif. Alors qu'en termes de système de jeu, la macrostructure de la bifurcation se résume en une variation du trajet d'Elwin sur la carte du monde, les différences topographiques des maps entre elles sont vectrices d'une bifurcation narrative à part entière.

À partir du cas de Der Langrisser, si l'on désire mieux comprendre l'articulation entre les mécanismes axiomatiques et narratifs de la bifurcation, il convient de réfléchir plus avant à la portée narrative du géographique et du topographique dans le T-RPG. Pour ce faire, je propose à présent de mettre en regard la construction diégétique de Tactics Ogre: Lets Us Cling Together avec celle de Der Langrisser.

\section{La map : discriminant spatial au service de la bifurcation narrative?}

Si l'on compare l'architecture de Der Langrisser avec celle de Tactics Ogre (pourtant considérée comme emblématique), les trois lignes d'intrigue de Tactics Ogre (correspondant aux alignements moraux conventionnels du RPG à la Donjons \& Dragons : Loyal, Chaotique, Neutre) convergent toutes vers un même chapitre final ne reposant que sur deux fins alternatives, dépendant quasi exclusivement du personnage de Kachua (en fonction de la survie de cette dernière au cours de la partie). ${ }^{7}$ Cependant, en dépit de cette différence flagrante dans la structure non-linéaire des deux jeux, il faut tout de même convenir d'une certaine proximité entre Der Langrisser et Tactics Ogre. Dans les deux cas, chaque ligne narrative correspond à un enchaînement spécifique de maps, camouflé derrière un choix présenté comme moral. Si l'on prend l'exemple de Tactics Ogre, le choix de rester loyal au duc Ronway fait accéder le joueur à un chapitre 2 composé de neuf maps allant de la ville de Baramus aux salles du château d'Amorika. En revanche, si le joueur choisit de s'opposer aux ordres du duc en refusant de massacrer les habitants de Baramus, il entame un parcours à travers les douze maps de la version « chaotique » du chapitre 2, allant cette fois-ci d'Ashton au château d'Amorika. Si la plupart des maps sont communes aux deux routes, il ne s'agit pourtant pas toujours des mêmes configurations spatiales.

Ainsi, la ville de Rime revêt-elle deux apparences distinctes selon le choix du joueur. Dans la route "loyale", la map est exclusive au chapitre dans le sens où elle diffère de la version standard utilisée pour les combats aléatoires. Dans la route "chaotique», le combat contre Zapan se déroule sur la map générique de Rime. La map de la version « loyale » est une reprise légèrement modifiée de celle que le joueur a déjà pu rencontrer lors de la bataille obligatoire du chapitre 1 . Il s'agit d'un paysage urbain, avec une forte 
déclivité et une série d'habitations concentrées dans la partie droite de l'écran. Ce déséquilibre architectural entre les parties gauche et droite de la map accentue l'effet miroir entre les deux frères ennemis que sont Denam et Vice. Cela prépare également l'issue de la confrontation, c'est-à-dire le moment où chacun des personnages va partir dans une direction opposée. À l'inverse, la route « chaotique » reprend la map du cœur de la ville de Rime afin de pouvoir faire un usage narratif et tactique de l'espace supérieur. Protégé par une muraille de bâtiments, Zapan - le chef ennemi - bénéficie d'un avantage sur l'armée du joueur. De plus, cette cour intérieure fait office de théâtre au piège ourdi par Zapan, ainsi qu'à l'enlèvement de Kachua. Si la bifurcation narrative ne modifie pas drastiquement le parcours du joueur à travers la géographie fictionnelle du monde de Tactics Ogre (l'aspect stratégique, pour reprendre ce terme du wargame), les variantes spatiales de Rime présentes dans chacune des lignes narratives utilisent les éléments topographiques de l'espace isométrique comme des ressorts narratifs. Jouant le rôle de véritables discriminants spatiaux, ces maps alternatives sont le lieu où se noue la divergence diégétique à l'intérieur de la narration vidéoludique.

Dans la mise en géographie de leur univers respectif, Der Langrisser et Tactics Ogre répondent à des logiques différentes. Dans Der Langrisser, tous les déplacements sur la carte du monde sont irréversibles. Le joueur n'est que le spectateur des déplacements d'Elwin, alors que Tactics Ogre laisse son joueur décider du lieu dans lequel Denam se rendra. En ce sens, il apparaît de manière évidente que le géographique ne constitue en rien le cœur générique du T-RPG. Bien que cela puisse varier selon le gameplay retenu, la carte $\mathrm{du}$ monde joue principalement un rôle d'illustration, contrairement au topographique qui n'est pas confiné à un tel rôle. Que ce soit dans Der Langrisser ou dans Tactics Ogre, on retrouve un usage similaire de l'espace isométrique. Dans les deux cas, les maps ont une fonction narrative majeure. Elles s'inscrivent à la fois dans un déroulé chronologique (la succession des maps, représentant chacune un chaînon diégétique), mais également dans un réseau transversal né des bifurcations de l'intrigue. Ce réseau peut être qualifié d'intertextuel (en prenant l'idée de texte dans son acception élargie), mais l'on pourrait aussi le désigner comme intertopique (à partir du sémantisme spatial du grec topos).

29 Parce que les bifurcations de ces T-RPGs en font des mondes foisonnants, il s'agit de système ludique dont il est difficile de faire l'expérience totale. Mais, contrairement aux mondes ouverts, il est en soi possible de parvenir à cette totalité. C'est pour cette raison qu'il est important de reconnaître la diversité des liens sur lesquels se fonde la construction de ces T-RPGs. La causalité linéaire n'est pas la seule forme logique à gouverner la narrativité de ces jeux. À travers les relations de similitudes et de différences liant chaque map à leurs alter ego, la topographie devient le moteur d'une dynamique narrative faisant s'entrechoquer la pluralité des espaces-temps constituant l'intrigue de ces T-RPGs.

\section{Entre temps et espace : considérations transmédiatiques au sujet de la bifurcation narrative}

Dès lors, en dépit de la valeur spatiale de l'embranchement diégétique, ce dernier contribue - d'une façon paradoxale - à une re-temporalisation de l'intrigue du T-RPG. Pour mieux comprendre cette ambivalence narrative, il demeure pertinent d'aborder la question de la bifurcation selon un angle transmédiatique. Dans sa célèbre nouvelle Le 
Jardin aux sentiers qui bifurquent (1941), Jorge Luis Borges propose une rêverie théorique sur ce que recouvre la séparation de la diégèse en une multitude de possibles :

Dans toutes les fictions, chaque fois que diverses possibilités se présentent, l'homme en adopte une et élimine les autres; dans la fiction du presque inextricable Ts'ui Pên, il les adopte toutes simultanément. Il crée ainsi divers avenirs, divers temps qui prolifèrent ainsi et bifurquent (Borges, 1941, p. 193).

À partir de l'exemple fictif du livre de Ts'ui Pên, Borges montre comment, grâce à la prolifération des récits, le temps et l'espace se fécondent mutuellement. Si le principe de la bifurcation est bien d'essence spatiale (dans l'espace, tout est donné simultanément), il permet au temps de se multiplier. Autrement dit, lorsque le jardin bifurque, il s'ouvre à la temporalité. Il fait émerger en son sein une pluralité de futurs. Dans la fiction littéraire, l'espace de la bifurcation est porteur de temps.

Il en va exactement de même dans Der Langrisser et dans Tactics Ogre. Dans Tactics Ogre, lorsque le joueur contrôlant Denam choisit de massacrer les habitants de Baramus, il prend un chemin dans les deux sens du terme: il va traverser un parcours de maps spécifiques, tout en avançant vers un dénouement du récit dont il reconnait la singularité parmi les multiples possibles de l'intrigue. On pourra ici objecter que le joueur naïf n'a pas forcément conscience de la portée de son choix. Entre le début du jeu et la fin du chapitre 1, le joueur a déjà dû faire de nombreux choix sans que ces derniers ne paraissent avoir une quelconque incidence narrative. Pourquoi en serait-il autrement dans ce cas? Sans connaissance du scénario de Tactics Ogre, comment le joueur peut-il savoir que ce choix est déterminant pour la suite? Après tout, le dénouement du chapitre 1 ne pourrait-il pas aussi bien résulter de la somme des choix précédents? Cependant, comme dans Le Jardin aux sentiers qui bifurquent, les bifurcations du T-RPG ne s'abolissent pas entre elles. Chaque choix ouvre le possible d'une nouvelle partie, d'une autre expérience de jeu dans laquelle ce sont les autres alternatives qui pourront être explorées. Face à des situations potentiellement sources d'une bifurcation narrative, le joueur projette "divers avenirs ", qui seront ou non actualisés dans la réalité. Par le prisme de Denam ou d'Elwin, aussi bien dans Tactics Ogre que dans Der Langrisser, le parcours spatial à travers la diégèse s'inscrit dans une trajectoire de vie (fictionnelle), favorisant la saturation du récit.

Bien évidemment, le joueur dispose de la liberté de traiter ces jeux comme toutes les autres fictions : d'adopter - même involontairement - une possibilité, et d'éliminer toutes les autres. Dans le labyrinthe, le voyageur peut choisir de ne pas se confronter à l'expérience de l'inextricable, de considérer qu'il n'y a effectivement qu'un seul chemin à suivre. Mais, pour les aventuriers audacieux, il est également possible de devenir partie prenante de l'enchaînement a-narratif entre les maps. Cela se traduit alors par la constitution de relations intertopiques à valeur péri-narrative. ${ }^{9}$ La relation causale perd ici sa préséance : elle n'est plus ce qui préside exclusivement à l'expérience de jeu, même si elle continue de structurer l'expérience $d u$ jeu.

34 Appliqué au jeu vidéo, le modèle proposé par Borges dans Le Jardin aux sentiers qui bifurquent permet de rendre compte des mécanismes liant la non-linéarité du système de jeu à la non-linéarité narrative. En raison de la nature même du T-RPG, la non-linéarité du système de jeu prend obligatoirement la forme de variations spatiales. Or, la narrativité non-linéaire requiert une dispersion des temporalités. Si les bifurcations spatiales créent le temps, le système de jeu du T-RPG induit - de facto - la création de temporalités narratives non-linéaires. Mais, et il s'agit là du génie de Borges, la pluralisation de 
l'intrigue ne présuppose alors pas nécessairement la pluralité des expériences. Dans l'analyse des bifurcations de Der Langrisser ou de Tactics Ogre, on se heurte constamment aux modalités de l'expérience de jeu. Le joueur connaît-il la bifurcation? Fait-il un choix volontaire? Cette question de l'intention est toujours présente en filigrane, comme elle l'est lorsque l'on étudie la place du lecteur dans les littératures non-linéaires. Ce dont témoigne le propos borgèsien, c'est précisément du fait que l'architecture spatiotemporelle de l'œuvre fictionnelle est une réalité (que l'on pourra dire objective), tandis que l'expérience empirique de l'espace-temps fictionnel en constitue une autre (subjective). En fonction du médium, l'articulation entre ces deux niveaux ne se fera pas de la même manière. Comme présenté plus haut, la topographie de l'espace isométrique remplit précisément ce rôle dans le T-RPG. Tout en participant à la séquence causale de l'expérience de jeu, elle favorise la mise en réseau totalisante des maps entre elles. De la même manière que dans "la fiction du presque inextricable Ts'ui Pên ", cette double dynamique (d'espace et de temps) lie les deux espaces-temps de l'expérience de la fiction. En cela, elle fonde - dans le même temps - l'expérience de jeu des T-RPGs non-linéaires.

\section{Conclusion}

Dans sa structuration même, le $T-R P G$ constitue un objet narratif bien singulier. Fondé sur une - ou plusieurs - succession de maps, il se révèle foncièrement topologique. Dans la mesure où il instaure un primat des catégories spatiales, il atténue les ressorts temporels habituellement associés à la narration. Des critiques comme Marie-Laure Ryan (2016) se sont déjà penchés sur la manière dont l'espace tend à coloniser les mécanismes de la narration, et ce quel que soit le médium. Ainsi, lorsqu'elle porte sur les catégories de temps et d'espace (que Kant posait comme des a priori de l'expérience), la narratologie peut-elle constituer un outil transmédiatique, du texte au jeu vidéo et vice-versa?

Néanmoins, il s'avère également que l'axiomatique propre à tout médium ludique rend ambivalente la nature de la temporalité dans le jeu vidéo. Constamment, le temps du récit entre en concurrence avec le temps de jeu (celui qui est vécu par le joueur). En raison de cette co-existence entre deux temporalités distinctes, le jeu vidéo doit chercher à faire coïncider ces deux structures temporelles, - au risque que l'axiomatique ne vienne invalider des pans entiers du récit. Les bifurcations narratives propres au T-RPG des années 1990-1995 constituent donc des objets propices à l'exploration narratologique de ce «disnarrated» qu'évoque Baroni (2016). Lorsque les récits se démultiplient, le jeu vidéo ouvre au joueur un vaste champ dans lequel l'expérience empirique du jeu devient elle-même la dynamique de la narration.

\section{BIBLIOGRAPHIE}

BARONI R. (2007), La Tension narrative. Suspense, curiosité et surprise, Paris, Seuil. 
BARONI R. (2016), «The Many Ways of Dealing with Sequence in Contemporary Narratology », in R. Baroni \& F. Revaz (dirs.), Narrative Sequence in Contemporary Narratology, Columbus, Ohio State University Press, pp. 1-7.

BARONI R. (2017), «The Garden of Forking Paths : Virtualities and Challenges for Contemporary Narratology ", in P. K. Hansen, J. Pier, P. Roussin, W. Schmid (dirs.), Emerging Vectors of Narratology, Berlin, De Gruyter, pp. 247-264.

BORGES J. L. (1941), Fictions, Paris, Gallimard, 1994.

CAÏRA O. (2016), « Théorie de la fiction et esthétique des jeux », Sciences du jeu, n 6. http:// journals.openedition.org/sdj/671

JENKINS H. (2002), « Game design as narrative architecture », in P. Harrington \& N. Frup-Waldrop (dirs.), First Person : New Media As Story, Performance, and Game, Cambridge, MIT Press, pp. 118-130.

LAVOCAT F. (2016), Fait et fiction. Pour une frontière, Paris, Seuil.

MARTI M. (2014), « La narrativité vidéoludique : une question narratologique », Cahiers de narratologie, $\mathrm{n}^{\circ} 27$. http://journals.openedition.org/narratologie/7009

PETERSON J. (2012), Playing at the Worlds, San Diego, Unreason Press.

RICHARDSON B. (2016), « Unusual and Unnatural Narrative Sequences », in R. Baroni \& F. Revaz (eds), Narrative Sequence in Contemporary Narratology, Columbus, Ohio State University Press, pp. 163-175.

RYAN M.-L. (2016), « Sequence, Linearity, or Why Be Afraid of Fixed Narrative Order ? », in R. Baroni \& F. Revaz (dirs.), Narrative Sequence in Contemporary Narratology, Columbus, Ohio State University Press, pp. 176-193.

STERN C. (2012), « Why categorize jRPGs and wRPGs? », IndieRPGs.com. http:// indierpgs.com/2012/03/why-categorize-jrpgs-and-wrpgs/

\section{NOTES}

1. Des illustrations présentant ces différents modes de jeu sont disponibles sur la page dédiée à Ultima III du site hardcoregaming101.net : http://www.hardcoregaming101.net/ultima/ultima4.htm 2. On peut considérer la réaction de l'équipe d'Ultima Codex (fansite consacré à la série Ultima) face à l'article de Craig Stern : http://ultimacodex.com/2012/03/why-do-we-separate-westernand-japanese-rpgs/

3. Pour plus d'informations, on se référera au guide publié sur le site gamefaqs.com: https:// www.gamefaqs.com/nes/562649-fire-emblem-ankoku-ryu-to-hikari-no-tsurugi/faqs/21975

4. Je reprends ici le terme « axiomatique » dans son opposition avec le versant purement narratif (car mimétique) du jeu. L'axiomatique renvoie donc à la résolution de problèmes, inhérente à toute activité ludique (Caïra, 2016).

5. Cette difficulté qu'a la distinction fabula/sjuzhet à rendre compte des mécanismes liés à l'interactivité vaut également pour la narratologie littéraire (Richardon, 2016).

6. Pour preuve, on consultera cette représentation graphique de la structure narrative de Der Langrisser, disponible sur le site gamefaqs.com: https://www.gamefaqs.com/snes/588277-derlangrisser/faqs/48923

7. Pour référence, on consultera le site luct.tacticsogre.com, dédié à la version SNES du jeu : http:// luct.tacticsogre.com/valeria.html 
8. Je me place ici dans la continuité de l'interprétation métanarrative du Jardin aux sentiers qui bifurquent que l'on trouve chez Baroni (2017).

9. J'emploie le terme " péri-narratif » comme un adjectif renvoyant à l'action du joueur lorsqu'il ajoute sa propre interprétation des événements au fil pré-structuré de l'intrigue (Jenkins, 2002 ; Marti, 2014).

\section{RÉSUMÉS}

En tant que mélange entre jeu de rôle et jeu de stratégie au tour par tour, le Tactical RPG ou « jeu de rôle tactique » apparaît comme un objet hybride devant concilier la tension narrative propre au jeu de rôle et le fonctionnement purement a-diégétique des échecs. Me focalisant sur la période 1990-1995, je propose pour étudier cette hybridité un parcours analytique allant du premier Fire Emblem à la sortie de Tactics Ogre : Let Us Cling Together en octobre 1995. J'étudie ainsi la manière dont le système de jeu du T-RPG met l'espace ludique isométrique en géographie afin d'en faire le cœur d'une dynamique narrative, où l'espace et le temps interviennent de concert. Par l'entremise du cas spécifique de la bifurcation narrative, cet article entend contribuer à la réflexion en cours sur la possibilité de pratiquer une narratologie transmédiatique.

As it crosses borders between role-playing games and turn-based strategy games, Tactical RPG seems to be a hybrid object, which has to reconcile the narrative tension one can find in role playing games, and the a-diegeticness of chess. I propose studying such hybridity by focusing on the years 1990-1995, from the first Fire Emblem to October 95, i.e. the release of Tactics Ogre. As I study the way T-RPG turns the isometric squares of the ludic space into a true geographic world, I bring out the fact this geographical turn plays the main role in the spatial and temporal dynamics of the narrative. By considering the specific case of forking paths in narration, this paper wished to points towards the possibility of a transmediatic narratology.

\section{INDEX}

Mots-clés : jeu de rôle tactique, espace, temps, non-linéarité, bifurcation narrative

Keywords : tactical rpg, time, space, non-linearity, forking paths

\section{AUTEUR}

\section{SÉBASTIEN WIT}

Université Paris Nanterre 